\title{
Factores asociados a periodontitis crónica en una población obesa: un estudio de corte transversal
}

\author{
Factors associated with chronic periodontitis in an obese population: \\ a cross-sectional study
}

\author{
Silie Arboleda ${ }^{1,2}$, Sandra Sánchez ${ }^{2}$, Katherine Flórez², Vanessa Figueroa ${ }^{2}$, Gloria Inés Lafaurie ${ }^{2,3}$, \\ Fanny Aldana-Parra ${ }^{4}$, Carlos Felipe Chaux ${ }^{4}$
}

Recibido: 30 de abril de 2018. Aceptado para publicación: 26 de Junio de 2018 https://doi.org/10.35454/rncm.v1n2.040

\section{Resumen}

Introducción: la obesidad es una enfermedad crónica multifactorial que ha sido positivamente asociada a la enfermedad periodontal. El propósito de este estudio fue analizar los factores asociados a periodontitis crónica en un grupo de pacientes obesos.

Métodos: este estudio evaluó 101 pacientes obesos con edades entre los 18 y 60 años que asistieron a un centro de referencia para el tratamiento quirúrgico de la obesidad. A los participantes se les tomaron medidas antropométricas, se les realizó un examen periodontal y se completó un cuestionario estructurado. La obesidad fue definida como un índice de masa corporal $(I M C) \geq 30 \mathrm{~kg} / \mathrm{m}^{2}$. La periodontitis fue definida de acuerdo con los criterios de Eke \& Page, 2012. Se realizaron análisis de tipo descriptivo, bivariado y multivariado.

Resultados: la prevalencia de periodontitis en este grupo de pacientes obesos fue de $35,6 \%$. Respecto a la severidad $19,8 \%$ de los pacientes tuvieron periodontitis crónica leve. El $44,5 \%$ de los sujetos fueron clasificados como obesos tipo I, 38,6\% obesos tipo II y $16,8 \%$ obesos tipo III. La periodontitis crónica estuvo asociada en forma significativa a la edad, los años de educación y a la hipertensión arterial. Después de ajustar por algunas variables, solo la edad (46-60 años) permaneció significativamente asociada al desarrollo de periodontitis [OR: 4,91, IC $95 \%$ 1,02-23,56].

Conclusión: se encontró una prevalencia de periodontitis crónica de 35,6\%. El incremento en la edad, $\leq 12$ años de educación y la hipertensión son factores de riesgo para la aparición de periodontitis en este grupo de pacientes.

Palabras clave: periodontitis crónica, epidemiología, índice de masa corporal, obesidad, prevalencia.

Unidad de Investigación Epidemiológica Clínica Oral (UNIECLO), Universidad El Bosque, Bogotá, D.C, Colombia.

2 Facultad de Odontología, Posgrado de Periodoncia y Medicina Oral, Universidad El Bosque, Bogotá, D.C, Colombia.

Correspondencia: Silie Arboleda

siliesoad@gmail.com

\section{Summary}

Background: Obesity is a multifactorial chronic disease that has been associated positively with periodontal disease. The purpose of this study was to analyze the associated factors to chronic periodontitis in a group of obese patients.

Methods: This study evaluated 101 obese participants aged between 18 and 60 who attended a referral center for the surgical treatment of obesity. All participants underwent periodontal examination, had anthropometric measurements and completed the structured questionnaire. Obesity was defined as a body mass index $(\mathrm{BMI}) \geq 30 \mathrm{~kg} / \mathrm{m}^{2}$. Periodontitis was defined according to the criteria of Eke \& Page, 2012. Descriptive analysis, bivariate and multivariate were used.

Results: The prevalence of periodontitis in this group of obese patients was $35.6 \%$. Regarding the severity, $19.8 \%$ of the patients had mild chronic periodontitis. $44.5 \%$ of the subjects were classified as obese type I, $38.6 \%$ obese type II and $16.8 \%$ obese type III. Chronic periodontitis was significantly associated in a positive way with age, years of education and arterial hypertension. After adjusting for some variables, only age (46 - 60 years) remained significantly associated with increased odds of periodontitis [OR: 4.91, $95 \% \mathrm{Cl} 1.02$ - 23.56].

Conclusion: A prevalence of chronic periodontitis of $35.6 \%$ was found. The increase in age, $\leq 12$ years of education and hypertension are risk factors for the manifestation of periodontitis in this group of patients.

Keywords: Chronic periodontitis; Epidemiology; Body mass index; Obesity; Prevalence.

\footnotetext{
3 Unidad de Investigación Básica Oral (UIBO), Universidad El Bosque, Bogotá, D.C, Colombia.

4 Cirugía para la Obesidad. Bogotá, D.C, Colombia.
} 


\section{INTRODUCCIÓN}

Las enfermedades crónicas no transmisibles constituyen un importante problema de salud pública en el mundo ${ }^{(1)}$. La obesidad definida por la Organización Mundial de la Salud (OMS) como un Índice de Masa Corporal $(\mathrm{IMC}) \geq 30 \mathrm{~kg} / \mathrm{m}^{2(2,3)}$, se considera una enfermedad crónica multifactorial que surge de una excesiva acumulación de grasa, resultante de la interacción entre el genotipo y el medio ambiente ${ }^{(4)}$.

En el ámbito mundial, entre 1980 y 2013, la prevalencia de sobrepeso y obesidad se ha incrementado en $27,5 \%$ en la población adulta en especial en la mayoría de los países desarrollados y en desarrollo ${ }^{(1)}$. En 2016, alrededor de $13 \%$ de la población adulta global (11\% hombres y $15 \%$ mujeres) presentaba obesidad ${ }^{(3)}$.

En Colombia, según la Encuesta Nacional de la Situación Nutricional (ENSIN) uno de cada tres jóvenes y adultos tiene sobrepeso $(37,7 \%)$, mientras que uno de cada cinco presenta obesidad (18,7\%). En este sentido, $56,4 \%$ de la población adulta registra exceso de peso, lo que significa un incremento de 5,2 puntos porcentuales con respecto a 2010. La obesidad es más frecuente en mujeres $(22,4 \%)$ que en hombres $(14,4 \%)^{(5)}$.

La obesidad constituye un factor de riesgo para el desarrollo de hipertensión, diabetes mellitus tipo 2, infarto, dislipidemias y algunos tipos de cáncer ${ }^{(6)}$. Evidencia publicada recientemente ha sugerido una asociación entre la obesidad y la periodontitis, dos enfermedades clasificadas por la OMS como crónicas no transmisibles y mejor referenciadas a través de un enfoque de factor de riesgo común debido a las múltiples causas implicadas en su inicio y progresión ${ }^{(7,8)}$.

La periodontitis es una enfermedad inflamatoria crónica causada por la infección de los tejidos de soporte que rodean el diente ${ }^{(9)}$. La infección comienza con la colonización y el crecimiento de un pequeño grupo de bacterias Gram-negativas predominantemente anaerobias y espiroquetas, en particular Porphyromonas gingivalis, Tannerella forsythia y Treponema denticola ${ }^{(9,10)}$. En el ámbito mundial, la periodontitis afecta entre 20 $\%$ a $50 \%$ de la población general ${ }^{(11)}$. La susceptibilidad a la enfermedad es altamente variable y depende de la respuesta del huésped a los patógenos periodontales ${ }^{(9)}$. Aunque la causa primaria es bacteriana, la progresión y las características clínicas están influenciadas por factores genéticos y adquiridos que pueden modificar la susceptibilidad a la infección ${ }^{(12)}$.
La relación entre obesidad y periodontitis fue reportada por primera vez en 1977, cuando Perlstein y Bissada observaron cambios histopatológicos en el periodonto de ratas obesas sometidas a periodontitis inducida por ligadura ${ }^{(13)}$. En humanos, diversos estudios de corte transversal sugieren que la obesidad puede estar directamente asociada a la periodontitis ${ }^{(14-17)}$.

Durante los últimos años, revisiones sistemáticas y meta-análisis han resumido los resultados de los estudios que evalúan la asociación entre sobrepeso/ obesidad y periodontitis, encontrando una asociación positiva significante entre el estatus de la obesidad y la prevalencia de enfermedad periodontal ${ }^{(7,8,18,19)}$.

La plausibilidad biológica de esta asociación se basa en la producción de citoquinas proinflamatorias como el factor de necrosis tumoral $\alpha$ (FNT- $\alpha$ ) y la interleuquina-6 (IL-6) por parte del tejido adiposo $^{(20)}$, los cuales afectan el metabolismo corporal generando el desarrollo de una inflamación sistémica de bajo grado. Los niveles de estas citoquinas proinflamatorias son proporcionales al IMC, principalmente en individuos con obesidad abdominal; de tal manera que un incremento en la grasa corporal podría inducir una mayor respuesta inflamatoria en la enfermedad periodontal ${ }^{(20)}$.

La asociación entre estas dos condiciones constituye un tópico importante dado que ambas enfermedades comparten un componente inflamatorio y una alta prevalencia en la población adulta, que requieren interés por parte de la comunidad científica. El propósito del presente estudio fue analizar los factores asociados a la enfermedad periodontal en un grupo de pacientes obesos que asistieron a un centro de referencia para el tratamiento de la obesidad.

\section{MATERIALES Y MÉTODOS}

\section{Participantes}

Este estudio de corte transversal evaluó 101 pacientes obesos de un centro de referencia para el tratamiento quirúrgico de la obesidad en Bogotá, Colombia (Cirugía para la Obesidad), quienes iban a ser sometidos a cirugía bariátrica. A los participantes se les tomaron medidas antropométricas, se les realizó un examen periodontal y completaron un cuestionario. Los criterios de inclusión tuvieron en cuenta individuos con edades entre los $18 \mathrm{y}$ 60 años con un IMC $\geq 30 \mathrm{~kg} / \mathrm{m}^{2}$. Sujetos con menos de 12 dientes en la boca y que estuvieran sometidos a tratamiento de ortodoncia fueron excluidos. 


\section{Cuestionario}

Se diseñó un cuestionario estructurado que fue aplicado por dos entrevistadores entrenados. Se aplicó previamente una prueba piloto sobre 30 individuos para garantizar la claridad de las preguntas. Las características sociodemográficas incluidas fueron: sexo, edad, años de educación, estado civil y estrato socioeconómico. Indicadores de riesgo como sangrado, movilidad $y$ antecedentes de pérdida dental por aflojamiento fueron indagados por autoreporte. Dentro de los hábitos de higiene oral se registraron: frecuencia de cepillado, frecuencia de uso de seda dental y enjuague bucal. La historia médica incluyó antecedentes de diabetes mellitus e hipertensión por autoreporte. El tabaquismo se definió como fumador actual (ha fumado en el último mes), exfumador (ha fumado, pero lleva 6 meses sin hacerlo) y no fumador (nunca ha fumado). Cuando el paciente respondió ser fumador se preguntó por cuantos cigarrillos fumaba diariamente.

\section{Medidas antropométricas}

Las medidas antropométricas fueron tomadas según la recomendación de la OMS para la determinación de peso y talla en la población adulta ${ }^{(21)}$. El peso corporal fue tomado mediante una báscula de piso marca JCM referencia WSP 12 digital, con capacidad de 400 $\mathrm{kg}$ y precisión de $0,1 \mathrm{~kg}$ y la talla fue medida utilizando un estadiómetro portátil marca SECA 230 con escala hasta de $205 \mathrm{~cm}$ y precisión de $1 \mathrm{~mm}$. El IMC se calculó como la relación del peso en kilogramos sobre la talla en metros al cuadrado.

De acuerdo con las guías clínicas de la OMS, la obesidad fue definida para hombres y mujeres como un IMC $\geq 30 \mathrm{Kg} / \mathrm{m}^{2(2)}$. Los participantes fueron clasificados por el IMC como: obesos tipo I (IMC $\geq 30 \mathrm{Kg} / \mathrm{m}^{2}-34,9$ $\mathrm{Kg} / \mathrm{m}^{2}$ ), obesos tipo II (IMC $\geq 35 \mathrm{Kg} / \mathrm{m}^{2}-39,9 \mathrm{Kg}$ / $\mathrm{m}^{2}$ ) y obesos tipo III o mórbidos (IMC $\left.\geq 40 \mathrm{Kg} / \mathrm{m}^{2}\right){ }^{2,3}$

\section{Datos de los archivos médicos de los pacientes}

Información de salud como los niveles de glicemia basal y triglicéridos fueron obtenidos de los archivos médicos de los pacientes, estos exámenes de laboratorio fueron enviados durante la valoración previa a la cirugía bariátrica. Los valores de referencia fueron proporcionados por el laboratorio: de $70-100 \mathrm{mg} / \mathrm{dL}$ para la glicemia y menor a $150 \mathrm{mg} / \mathrm{dL}$ para los triglicéridos.

\section{Examen periodontal}

A los participantes se les realizó un examen periodontal completo, llevado a cabo por un periodoncista y dos estudiantes de periodoncia, quienes fueron previamente calibrados para controlar la variabilidad interexaminador. Se tomaron medidas de la profundidad de la bolsa (PB) y el nivel de inserción clínico (NIC) de 84 superficies dentales. El resultado del coeficiente de correlación intraclase (CCI) para la PB fue una medida promedio de 0,82 [IC $\% 0,72-0,88]$ y para el NIC fue 0,93 [IC \% 0,93 - 0,95] obteniendo una calibración con fuerza de concordancia buena para la $\mathrm{PB}$ y muy buena para el NIC. La condición periodontal excepto los terceros molares fue evaluada usando: índice de placa (IP), índice gingival (IG) (Löe \& Silness), PB y NIC. La presencia de placa bacteriana y sangrado fue evaluada en dos superficies por diente (vestibular y palatino/lingual). La placa fue registrada como 1 para placa visible luego de pasar la sonda por la superficie dental y 0 para ausencia de placa. El sangrado fue registrado 15 segundos después del sondaje. Se registró como 1 para presencia de sangrado y 0 ausencia de sangrado. La PB y el NIC se midieron en milímetros $(\mathrm{mm})$ en seis superficies por diente (mesio-vestibular, medio-vestibular, disto-vestibular, mesio-palatino/lingual, medio-palatino/lingual y disto-palatino/lingual) con una sonda periodontal Carolina del Norte No. 15 de marca Vitalcom. La PB se midió como la distancia entre el margen gingival a la parte inferior del surco gingival $^{(22)}$. El NIC se registró como la distancia que hay de la unión cemento-esmalte a la parte inferior del surco gingival $^{(22)}$. Para cada individuo los IP e IG fueron determinados como el porcentaje de sitios con placa y sangrado respecto al número total de sitios examinados. El promedio de la PB y NIC se calcularon a partir de las mediciones de la boca en seis sitios por diente. Se registró el número de dientes perdidos. Para la definición de la periodontitis se utilizaron los criterios de Eke \& Page (2012) comúnmente empleados por el Centro de Control de Enfermedades (CDC) y la Academia Americana de Periodoncia (AAP) ${ }^{(23)}$ (Tabla 1).

\section{Análisis estadístico}

Se utilizó el programa estadístico STATA versión 11 para procesar y analizar los datos. Las características individuales de los participantes fueron descritas utilizando distribución de frecuencias para variables categóricas, promedio y desviación estándar (DE) para 
Tabla 1. Definición de periodontitis propuesta por Eke \& Page, 2012

\begin{tabular}{|l|l|}
\hline \multicolumn{1}{|c|}{ Caso } & \multicolumn{1}{c|}{ Definición } \\
\hline Sin Periodontitis & Sin evidencia de periodontitis leve, moderada o severa. \\
\hline $\begin{array}{l}\text { Periodontitis crónica leve } \\
(\mathrm{PCL})\end{array}$ & $\begin{array}{l}\geq 2 \text { sitios interproximales con pérdida de inserción } \geq 3 \mathrm{~mm}, \mathrm{y} \geq 2 \text { sitios interproximales } \\
\text { con profundidad de bolsa } \geq 4 \mathrm{~mm} \text { (en diferente diente) } \mathrm{o} \text { un sitio con profundidad de } \\
\text { bolsa } \geq 5 \mathrm{~mm} .\end{array}$ \\
\hline Periodontitis crónica moderada (PCM) & $\begin{array}{l}\geq 2 \text { sitios interproximales con pérdida de inserción } \geq 4 \mathrm{~mm} \text { (en diferente diente), o } \geq 2 \\
\text { sitios interproximales con profundidad de bolsa } \geq 5 \mathrm{~mm} \text { (en diferente diente) }\end{array}$ \\
\hline Periodontitis crónica severa (PCS) & $\begin{array}{l}\geq 2 \text { sitios interproximales con pérdida de inserción } \geq 6 \mathrm{~mm} \text { (en diferente diente) y } \geq 1 \\
\text { sitio interproximal con profundidad de bolsa } \geq 5 \mathrm{~mm} .\end{array}$ \\
\hline
\end{tabular}

La periodontitis total se calculó como la suma de periodontitis leve, moderada y severa $\mathrm{mm}$ : milímetros

variables continuas cuando se comprobó el supuesto de normalidad, en caso contrario fueron descritas mediante medianas y rangos intercuartílicos. Para mirar la distribución de los datos se utilizó la prueba de Shapiro Wilk debido a que es una prueba consolidada y con potencia estadística. El test de $\mathrm{X}^{2}$ se utilizó para evaluar la asociación entre las variables categóricas.

La periodontitis fue definida como la variable dependiente y la obesidad como la variable independiente. OR crudos y ajustados y sus IC fueron calculados mediante un modelo de regresión logística no condicionado. Para la escogencia de las variables regresoras en el modelo multivariado se utilizó la técnica del stepwise con una probabilidad de entrada de 0,1 y de salida de 0,25 . Se ajustó por variables como: sexo, edad, años de educación, estrato e hipertensión. Las pruebas de hipótesis fueron consideradas estadísticamente significativas con un $p \leq 0,05$.

\section{CONSIDERACIONES ÉTICAS}

Este estudio fue aprobado por el Comité de Ética de la Universidad El Bosque. Los participantes fueron informados acerca de los objetivos del estudio, firmaron el consentimiento informado y decidieron participar de manera voluntaria. Las consideraciones éticas del presente estudio se ajustan a la Resolución 8430 de 1993, la cual establece las normas científicas, técnicas y administrativas para la investigación en Colombia.

\section{RESULTADOS}

Las características de la población de estudio se muestran en la Tabla 2. El estudio incluyó un total de 101 participantes ( 30 hombres y 71 mujeres) con edades entre los 18 y 60 años con un promedio de 37,0 y una DE de 11,8 . Del total de los sujetos evaluados $85,3 \%$ tenían más de 12 años de educación y $48,5 \%$ pertenecían a estratos socioeconómicos altos $(5,6)$.

Dentro de los indicadores de riesgo periodontal evaluados, $62,3 \%$ de los sujetos reportaron sangrado y $10,9 \%$ movilidad y pérdida dental por aflojamiento.

Respecto a hábitos de higiene oral, todos los participantes manifestaron cepillar sus dientes, de los cuales 54,4\% reportó una frecuencia de cepillado de 3 veces al día; $71,3 \%$ reportaron usar seda dental y $63,4 \%$ enjuague bucal.

En el cuestionario 7,9\% de los sujetos manifestaron ser diabéticos tipo II y 13,8 \% hipertensos. 14,9 \% de los sujetos fueron clasificados como fumadores actuales. Respecto al tipo de obesidad, 44,5\% de los sujetos fueron clasificados como obesos tipo I, 38,6 \% obesos tipo II y $16,8 \%$ obesos tipo III.

El promedio (DE) del IMC para este grupo de pacientes obesos fue 35,39 $(4,18)$. El promedio (DE) de la PB fue de 2,33 mm (0,35),y para el NIC fue $1,08 \mathrm{~mm}$ $(0,64)$, el porcentaje de superficies con sangrado fue $60,67 \%(20,55)$ y el de superficies con placa fue de $52,15 \%(22,78)$. La frecuencia de periodontitis para este grupo de pacientes fue $35,6 \%$. Respecto a la severidad, 19,8 \% de los pacientes tuvieron PCL, 6,9 \% PCM y $8,9 \%$ PCS.

No hubo diferencias estadísticamente significativas en el IP y el IG entre el grupo de pacientes que tuvieron periodontitis y los que no tuvieron.

La Tabla 3 muestra los resultados del promedio (DE) de los parámetros periodontales evaluados para los 3 grupos de obesidad. No existieron diferencias estadísti- 
Tabla 2. Características sociodemográficas, antropométricas y clínicas relevantes de los participantes

\begin{tabular}{|c|c|c|c|c|c|}
\hline \multicolumn{2}{|l|}{ Variable } & \multicolumn{2}{|c|}{ Periodontitis* } & \multirow{3}{*}{$\begin{array}{c}\text { Total } \\
101(100 \%)\end{array}$} & \multirow{3}{*}{$\begin{array}{l}\text { Valor } \mathbf{p} \\
0,2942^{*}\end{array}$} \\
\hline & & & & & \\
\hline Sexo & $\begin{array}{l}\text { Hombres } \\
\text { Mujeres }\end{array}$ & 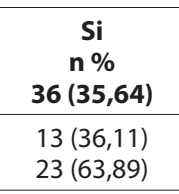 & $\begin{array}{c}\begin{array}{c}\text { No } \\
\text { n \% } \\
\mathbf{6 5}(\mathbf{6 4 . 3 6 )}\end{array} \\
17(26,15) \\
48(73,85) \\
\end{array}$ & & \\
\hline Edad (años) & Promedio (DE) & $\begin{array}{c}36 \text { sujetos } \\
42,36(12,14)\end{array}$ & $\begin{array}{c}65 \text { sujetos } \\
34,07(10,57)\end{array}$ & 101 sujetos & $0,0011^{* *}$ \\
\hline Años de educación & $\begin{array}{l}>12 \text { años } \\
\leq 12 \text { años }\end{array}$ & $\begin{array}{l}26(72,22) \\
10(27,78)\end{array}$ & $\begin{array}{c}60(92,31) \\
5(7,69)\end{array}$ & $\begin{array}{l}86(85,15) \\
15(14,85)\end{array}$ & $0,007 \dagger$ \\
\hline Estado civil & $\begin{array}{l}\text { Soltero } \\
\text { Casado } \\
\text { Viudo } \\
\text { Divorciado }\end{array}$ & $\begin{array}{c}13(36,11) \\
20(55,55) \\
1(2,78) \\
2(5,56)\end{array}$ & $\begin{array}{c}31(47,69) \\
31(47,69) \\
0(0) \\
3(4,62)\end{array}$ & $\begin{array}{c}44(43,56) \\
51(50,49) \\
1(0,99) \\
5(4,95)\end{array}$ & $0,226^{* * *}$ \\
\hline Estrato socioeconómico & $\begin{array}{l}\text { Alto (5-6) } \\
\text { Medio (3-4) } \\
\text { Bajo }(1-2)\end{array}$ & $\begin{array}{l}17(47,22) \\
17(47,22) \\
2(5,56)\end{array}$ & $\begin{array}{c}32(49,23) \\
32(49,23) \\
1(1,54)\end{array}$ & $\begin{array}{l}49(48,51) \\
49(48,51) \\
3(2,97)\end{array}$ & $0,600^{* * *}$ \\
\hline $\begin{array}{l}\text { Sangrado } \\
\text { (autoreporte) }\end{array}$ & $\begin{array}{l}\mathrm{No} \\
\mathrm{Si}\end{array}$ & $\begin{array}{c}6(16,67) \\
30(83,33) \\
\end{array}$ & $\begin{array}{l}32(49,23) \\
33(50,77) \\
\end{array}$ & $\begin{array}{l}38(37,62) \\
63(62,38) \\
\end{array}$ & $0,0012^{*}$ \\
\hline $\begin{array}{l}\text { Movilidad dental } \\
\text { (autoreporte) }\end{array}$ & $\begin{array}{l}\text { No } \\
\text { Si }\end{array}$ & $\begin{array}{l}25(69,44) \\
11(30,56)\end{array}$ & $\begin{array}{l}65(100) \\
0(0)\end{array}$ & $\begin{array}{l}90(89,11) \\
11(10,89)\end{array}$ & $0,0000^{*}$ \\
\hline $\begin{array}{l}\text { Pérdida dental por aflojamiento } \\
\text { (autoreporte) }\end{array}$ & $\begin{array}{l}\text { No } \\
\mathrm{Si}\end{array}$ & $\begin{array}{l}28(77,78) \\
8(22,22)\end{array}$ & $\begin{array}{c}62(95,38) \\
3(4,62)\end{array}$ & $\begin{array}{l}90(89,11) \\
11(10,89)\end{array}$ & $0,0065^{*}$ \\
\hline $\begin{array}{l}\text { Frecuencia de cepillado } \\
\text { (autoreporte) }\end{array}$ & $\begin{array}{c}3 \text { veces/día } \\
2 \text { veces/día } \\
1 \text { vez/día }\end{array}$ & $\begin{array}{c}19(52,78) \\
15(41,67) \\
2(5,56) \\
\end{array}$ & $\begin{array}{c}36(55,38) \\
26(40,00) \\
3(4,62) \\
\end{array}$ & $\begin{array}{c}55(54,46) \\
41(40,59) \\
5(4,95) \\
\end{array}$ & $1,000^{* * *}$ \\
\hline $\begin{array}{l}\text { Frecuencia de uso de seda } \\
\text { (autoreporte) }\end{array}$ & $\begin{array}{l}2 \text { veces/día } \\
1 \text { vez/día } \\
\text { Ninguna }\end{array}$ & $\begin{array}{c}(25,00) \\
16(44,44) \\
11(30,56) \\
\end{array}$ & $\begin{array}{l}23(35,39) \\
24(36,92) \\
18(27,69) \\
\end{array}$ & $\begin{array}{l}32(31,68) \\
40(39,60) \\
29(28,71) \\
\end{array}$ & $0,553+$ \\
\hline $\begin{array}{l}\text { Frecuencia de uso de enjuague } \\
\text { (autoreporte) }\end{array}$ & $\begin{array}{l}3 \text { veces (día) } \\
2 \text { veces (día) } \\
1 \text { vez (día) } \\
\text { Ninguna }\end{array}$ & $\begin{array}{c}1(2,78) \\
5(13,89) \\
20(55,56) \\
10(27,78)\end{array}$ & $\begin{array}{c}6(9,23) \\
8(12,31) \\
24(36,92) \\
27(41,54)\end{array}$ & $\begin{array}{c}7(6,93) \\
13(12,87) \\
44(43,56) \\
37(36.63)\end{array}$ & $0,246^{* * *}$ \\
\hline $\begin{array}{l}\text { Diabetes mellitus } \\
\text { (autoreporte) }\end{array}$ & $\begin{array}{c}\mathrm{No} \\
\mathrm{Si}\end{array}$ & $\begin{array}{c}30(83,33) \\
6(16,67)\end{array}$ & $\begin{array}{c}61(93,85) \\
4(6,15)\end{array}$ & $\begin{array}{l}91(90,10) \\
10(9,90)\end{array}$ & $0,160^{* * *}$ \\
\hline Hipertensión arterial (autoreporte) & $\begin{array}{l}\text { No } \\
\text { Si }\end{array}$ & $\begin{array}{l}26(72,22) \\
10(27,78)\end{array}$ & $\begin{array}{c}61(93,85) \\
4(6,15) \\
\end{array}$ & $\begin{array}{l}87(86,14) \\
14(13,86)\end{array}$ & $0,003+$ \\
\hline $\begin{array}{l}\text { Tabaquismo } \\
\text { (autoreporte) }\end{array}$ & $\begin{array}{c}\text { No fumador } \\
\text { Ex fumador } \\
\text { Fumador actual }\end{array}$ & $\begin{array}{c}27(75,00) \\
4(11,11) \\
5(13,89) \\
\end{array}$ & $\begin{array}{c}50(76,92) \\
5(7,69) \\
10(15,38)\end{array}$ & $\begin{array}{c}77(76,24) \\
9(8,91) \\
15(14,85)\end{array}$ & $0,876^{* * *}$ \\
\hline IMC & Promedio (DE) & $35,33(4,47)$ & $35,43(4,05)$ & 101sujetos & $0,7363^{* *}$ \\
\hline Glicemia & Promedio (DE) & $100,32(17,13)$ & $98,41(18,29)$ & 91 observaciones & $0,5105^{* *}$ \\
\hline Triglicéridos & Promedio (DE) & $150,86(55,30)$ & $140,54(54,08)$ & 88 observaciones & $0,4435^{* *}$ \\
\hline PB & Promedio (DE) & $2,53(0,46)$ & $2,22(0,22)$ & 101 sujetos & $0,0001^{* *}$ \\
\hline $\mathrm{NIC}$ & Promedio (DE) & $1,55(0,51)$ & $0,82(0,56)$ & 101 sujetos & $0,0000^{* *}$ \\
\hline Sangrado (\%) & Promedio (DE) & $63,5(20,06)$ & $59,10(20,81)$ & 101 sujetos & $0,3061 \neq$ \\
\hline Placa bacteriana (\%) & Promedio (DE) & $54,77(23,22)$ & $50,60(22,59)$ & 101 sujetos & $0,3927 \neq$ \\
\hline $\begin{array}{l}\text { Frecuencia de PC \% (n) } \\
\text { PCL } \\
\text { PCM } \\
\text { PCS }\end{array}$ & & $\begin{array}{c}35,64(36) \\
19,80(20) \\
6,93(7) \\
8,91(9)\end{array}$ & & & \\
\hline
\end{tabular}

DE: desviación estándar

PCL: periodontitis crónica leve, PCM: periodontitis crónica moderada, PCS: periodontitis crónica severa

*Diferencias calculadas mediante prueba $Z$ de diferencia de proporciones

**Diferencias calculadas mediante suma de rangos de Wilcoxon

***Diferencias calculadas mediante prueba exacta de Fisher

tDiferencias calculadas mediante prueba de $\mathrm{X}^{2}$

‡Diferencias calculadas mediante prueba $t$ student 
Tabla 3. Condición periodontal de los pacientes respecto al tipo de obesidad según el IMC

\begin{tabular}{|l|c|c|c|c|}
\hline \multicolumn{1}{|c|}{ Variable } & $\begin{array}{c}\text { Tipo I } \\
\text { (IMC } \mathbf{3 0 - 3 4 , 9 )} \\
\mathbf{n = 4 5} \\
\text { promedio } \pm \text { DE }\end{array}$ & $\begin{array}{c}\text { Tipo II } \\
\text { (IMC 35 - 39,9) } \\
\mathbf{n = 3 9} \\
\text { promedio } \pm \text { DE }\end{array}$ & $\begin{array}{c}\text { Tipo III } \\
\text { (IMC 40 - 49,9) } \\
\mathbf{n = 1 7} \\
\text { promedio } \pm \text { DE }\end{array}$ & Valor $\boldsymbol{p}$ \\
\hline IMC $\left(\mathrm{kg} / \mathrm{m}^{2}\right)$ & $31,61 \pm 1,29$ & $36,74 \pm 1,46$ & $42,33 \pm 2,04$ & $0,001^{*}$ \\
\hline Glicemia (mg/dL) & $95,92 \pm 15,97$ & $101,51 \pm 20,01$ & $100,87 \pm 16,59$ & 0,279 \\
\hline Triglicéridos & $142,53 \pm 63,35$ & $143,97 \pm 50,95$ & $148,06 \pm 39,47$ & 0,710 \\
\hline Bolsa periodontal $(\mathrm{mm})$ & $2,31 \pm 0,32$ & $2,27 \pm 0,26$ & $2,54 \pm 0,54$ & 0,134 \\
\hline Nivel de inserción (mm) & $0,98 \pm 0,59$ & $1,15 \pm 0,72$ & $1,20 \pm 0,59$ & 0,232 \\
\hline Sangrado (\% superficies) & $58,8 \pm 21,9$ & $62,6 \pm 19,7$ & $61,0 \pm 19,2$ & 0,647 \\
\hline Placa (\% superficies) & $54,3 \pm 22,5$ & $53,1 \pm 22,0$ & $44,2 \pm 24,6$ & 0,413 \\
\hline Dientes perdidos & $2,31 \pm 2,44$ & $3,66 \pm 4,34$ & $2,23 \pm 2,46$ & 0,673 \\
\hline Frecuencia de periodontitis $\mathrm{n}(\%)$ & $15(41,67)$ & $14(38,89)$ & $7(19,44)$ & 0,847 \\
\hline
\end{tabular}

IMC: índice de masa corporal

DE: desviación estándar

mg: miligramos, dL: decilitros, mm: milímetros

camente significativas para la PB (valor $p: 0,134)$, NIC (valor $p: 0,232$ ), porcentaje de placa (valor $p: 0,647)$ y porcentaje de sangrado (valor $p: 0,413$ ).

Como se muestra en la tabla 4, la periodontitis crónica fue significativamente asociada a la edad, los años de educación y la hipertensión arterial. Sujetos con edades entre 46 y 60 años tuvieron un OR de 8,53 (IC $95 \% 1,97$
-36,91) para tener periodontitis comparado con sujetos de 26 a 45 años $y \leq 25$ años. Tener $\leq 12$ años de educación fue asociado a un odds de tener periodontitis de 4,61 (IC $95 \% 1,43$ - 14,84) comparado con tener más de 12 años de educación. La hipertensión arterial fue asociada a un OR de tener periodontitis de 5,86 (IC $95 \%$ $1,68-20,41)$ comparado con sujetos no hipertensos.

Tabla 4. Modelo de regresión logística no condicionado para describir los factores asociados a periodontitis

\begin{tabular}{|c|c|c|c|c|c|}
\hline \multicolumn{2}{|c|}{ Variable } & \multicolumn{2}{|c|}{ Bivariado } & \multicolumn{2}{|c|}{ Multivariado* } \\
\hline & & OR (IC $95 \%)$ & Valor $p$ & OR (IC $95 \%)$ & Valor $p$ \\
\hline \multirow[t]{2}{*}{ Sexo } & Hombres & 1 & & 1 & \\
\hline & Mujeres & $0,62(0,26-1,50)$ & 0,296 & $0,86(0,31-2,34)$ & 0,769 \\
\hline \multirow[t]{3}{*}{ Edad (años) } & $\leq 25$ & 1 & & 1 & \\
\hline & $26-45$ & $2,32(0,59-9,04)$ & 0,223 & $2,35(0,56-9,18)$ & 0,244 \\
\hline & $46-60$ & $8,53(1,97-36,91)$ & $0,004^{*}$ & $4,91(1,02-23,56)$ & $0,046^{*}$ \\
\hline \multirow[t]{2}{*}{ Años de educación } & $>12$ años & 1 & & 1 & \\
\hline & $\leq 12$ años & $4,61(1,43-14,84)$ & $0,010^{*}$ & $2,94(0,80-10,8)$ & 0,104 \\
\hline \multirow{3}{*}{$\begin{array}{l}\text { Estrato } \\
\text { socioeconómico }\end{array}$} & Alto (V-VI) & 1 & & 1 & \\
\hline & Medio (III-IV) & $1(0,43-2,29)$ & 1 & $0,80(0,31-2,09)$ & 0,662 \\
\hline & Bajo (I-II) & $3,76(0,31-44,57)$ & 0,293 & $2,05(0,11-36,86)$ & 0,626 \\
\hline \multirow[t]{2}{*}{ Hipertensión arterial } & No & 1 & & 1 & \\
\hline & $\mathrm{Si}$ & $5,86(1,68-20,41)$ & $0,005^{*}$ & $2,91(0,72-11,73)$ & 0,131 \\
\hline
\end{tabular}

OR: odds ratio

IC: intervalo de confianza

*Ajustado por sexo, edad, años de educación, estrato socioeconómico e hipertensión arterial. 
En el análisis de regresión logística multivariado después de ajustar por algunas variables solo la edad permaneció significativamente asociada a una probabilidad de tener periodontitis de 4,91 (IC $95 \%$ 1,02 $-23,56)$.

\section{DISCUSIÓN}

La muestra de este estudio estuvo conformada por mujeres en $70,3 \%$, esto se justifica debido a las altas prevalencias de obesidad encontradas en el sexo femenino ${ }^{(24)}$ y también a que las mujeres tienden a someterse de manera más frecuente a cirugía bariátrica que los hombres. Estos hallazgos coinciden con lo reportado por Pataro en $2012^{(25)}$.

En este estudio, 35,6 \% de los participantes tuvieron periodontitis ( $36,1 \%$ en hombres y $63,8 \%$ en mujeres). Lo cual se encuentra en una fuerte discrepancia con los resultados reportados por el último Estudio Nacional de Salud Bucal (ENSAB IV) realizado en Colombia, quienes encontraron una prevalencia de periodontitis de $61,8 \%$. Ambos estudios emplearon el mismo criterio diagnóstico para la definición de la enfermedad periodontal (Eke \& Page, 2012) (23). $^{(2)}$

Respecto a la severidad el ENSAB IV reportó que la periodontitis moderada fue la más frecuente $(43,46 \%)$ (26). En nuestro estudio, fue más común la periodontitis leve con 19,8\% de los sujetos afectados.

La frecuencia de periodontitis crónica fue más baja de lo esperado, posiblemente porque la mayoría de los participantes presentaban buenos hábitos de higiene oral, un alto nivel educativo y pertenecían a estratos socioeconómicos altos.

Es probable que personas con un nivel socioeconómico más alto tengan actitudes más positivas con respecto a la higiene oral y el autocuidado, y un mejor acceso a los servicios de salud disponibles ${ }^{(27)}$. Todos los participantes de nuestro estudio manifestaron cepillar sus dientes, de estos $95 \%$ reportó cepillar sus dientes $\geq 2$ veces al día y más de la mitad de los sujetos reportó usar seda dental (71,3\%) y enjuague bucal $(63,4 \%)$. Una revisión sistemática reportó que un cepillado infrecuente ( $<1$ vez al día) constituye un factor de riesgo para enfermedad periodontal (OR: 1,41, IC $95 \% 1,25$ $-1,58)^{(28)}$. La AAP no ha identificado a la higiene oral como un factor preventivo para la enfermedad periodontal, pero atribuye la disminución de la prevalencia de gingivitis en Estados Unidos a unas buenas prácticas de higiene oral ${ }^{(29)}$.
Un estudio realizado en Brasil con una muestra de 345 sujetos encontró una prevalencia de periodontitis en población obesa de $66,9 \%{ }^{(25)}$. Ellos definieron la periodontitis según los criterios de Page \& Eke (2007) (22), los autores de este estudio utilizaron la misma definición, la cual fue actualizada por Eke \& Page en el $2012^{(23)}$, encontraron una frecuencia de periodontitis mucho más baja, tal vez por las características antes mencionadas de nuestros pacientes.

Khader en Jordania encontró una prevalencia de periodontitis en población obesa de $51,9 \%$, sin embargo, el criterio diagnóstico para la definición de la enfermedad periodontal fue diferente al empleado en nuestro estudio. La heterogeneidad en la definición de la periodontitis hace difícil la comparación de los resultados. Nosotros usamos el criterio del CDC/ AAP que es en la actualidad una de las definiciones más recomendadas para la vigilancia de la enfermedad periodontal ${ }^{(23)}$.

En este grupo de pacientes obesos no hubo diferencias significativas respecto a los IP e IG entre los pacientes con y sin periodontitis, lo cual indica que todos los pacientes presentaron niveles similares de inflamación gingival, estos hallazgos coinciden con de Castilhos et $a l$, quienes no encontraron una asociación significativa entre la presencia de bolsas periodontales y la obesidad, pero si entre la gingivitis y la obesidad ${ }^{(30)}$.

En nuestro estudio, el riesgo para periodontitis se aumenta 8,53 veces más (IC $95 \% 1,97$ - 36,91) para individuos con edades entre los 46 y 60 años. Esta variable presentó un intervalo de confianza amplio, lo que se puede explicar por el tamaño de muestra, pero mostró el valor $p$ más significativo. La edad ha sido asociada de manera significativa al desarrollo de enfermedad periodontal en diferentes poblaciones ${ }^{(26,31-33)}$. Un reciente estudio propone que 45 años sea el punto de corte para que los individuos sean evaluados respecto a las necesidades de tratamiento periodontal, como una importante estrategia de control de la periodontitis ${ }^{(34)}$.

Tener $\leq 12$ años de educación fue significativamente asociado a un incremento en la frecuencia de enfermedad periodontal de 4,6 veces comparado con sujetos con más de 12 años de educación. Un estudio multicéntrico realizado en América del Sur encontró que la presencia de inflamación gingival fue asociada de manera positiva a tener $\leq 12$ años de educación ${ }^{(27)}$. Diversos estudios han relacionado tener pocos años de educación con el desarrollo de periodontitis, lo cual confirma la consistencia de esta asociación ${ }^{(31,35)}$. 
La relación entre hipertensión y periodontitis ha sido reportada previamente ${ }^{(36)}$. Un estudio poblacional de corte transversal en Brasil reveló una asociación importante entre hipertensión y periodontitis en mujeres obesas ${ }^{(37)}$.

En el análisis multivariado, la edad fue la única variable que permaneció asociada al desarrollo de enfermedad periodontal. Es posible que debido al tamaño de muestra tan pequeño de pacientes con $\leq 12$ años de educación e hipertensión se hubiera perdido la significancia estadística en el odds de periodontitis en el modelo de regresión logística multivariado, por lo cual decidimos apoyarnos en el modelo bivariado, ya que las asociaciones encontradas se encuentran sustentadas en la literatura.

Del total de los participantes, $82,17 \%$ no presentaban comorbilidades tipo diabetes tipo 2 e hipertensión arterial, las cuales han sido reconocidas por la OMS como enfermedades crónicas no transmisibles relacionadas con la obesidad ${ }^{(3)}$, esto podría significar que estos pacientes presentaban un mejor metabolismo energético por lo que podría especularse que las citoquinas proinflamatorias como TNF- $\alpha$ e IL-6 relacionadas con ambas enfermedades no estuvieran críticamente elevadas, lo cual permitió encontrar una baja frecuencia de periodontitis respecto a la prevalencia en otras poblaciones obesas.

Diversos estudios longitudinales consideran que fumar constituye un factor de riesgo para periodontitis $^{(12)}$. En este estudio, no pudimos comprobar que el tabaquismo estuviera asociado al desarrollo de enfermedad periodontal, debido probablemente al tamaño de muestra tan bajo de individuos fumadores.

Dentro de las limitaciones de este estudio tenemos que algunas de las variables evaluadas dependían de los reportes dados por los pacientes, como los hábitos de higiene oral, el tabaquismo y otros. Los informes de los sujetos pueden no ser verdaderos o precisos algunas veces. Este estudio utilizó el IMC como único criterio para el diagnóstico de la obesidad, aunque el IMC es el indicador más usado debido a su facilidad de medición en estudios clínicos y epidemiológicos, puede implicar una clasificación errónea como resultado de una variación individual del tejido adiposo y la masa muscular $^{(25)}$. Se recomienda usar adicionalmente la medida de la circunferencia de la cintura, sobre la cual se establece que una medida $\geq 102 \mathrm{~cm}$ en hombres $y \geq 88 \mathrm{~cm}$ en mujeres corresponden al diagnóstico de obesidad abdominal, la cual es considerada un factor de riesgo para enfermedad cardiovascular ${ }^{(38)}$.
Otras limitaciones incluyen el tipo de estudio ya que los diseños transversales no permiten distinguir el orden temporal de los eventos y llegar a establecer una verdadera relación causal ${ }^{(39)}$. Un grupo control hubiera permitido establecer diferencias significativas entre la periodontitis en sujetos obesos y no obesos.

\section{CONCLUSIÓN}

Este estudio que representa una muestra de la población obesa colombiana presentó una prevalencia de enfermedad periodontal de 35,6\%, la cual se encontró asociada a factores como la edad, los años de educación y la hipertensión arterial.

\section{Agradecimientos}

A la Sociedad Cirugía para la Obesidad por su colaboración en el desarrollo de este estudio.

\section{Conflicto de intereses}

Ninguno.

\section{Financiación}

Este estudio fue financiado por la División de Investigaciones de la Universidad El Bosque.

\section{Referencias bibliográficas}

1. $\mathrm{Ng} \mathrm{M}$, Fleming $\mathrm{T}$, Robinson $\mathrm{M}$, et al. Global, regional, and national prevalence of overweight and obesity in children and adults during 1980-2013: a systematic analysis for the Global Burden of Disease Study 2013. Lancet. 2014;384:766-81.

2. Chopra A, Sivaraman K, Bhat SG. "United Pedicle Flap" for management of multiple gingival recessions. Journal of Indian Society of Periodontology. 2016;20:344-8.

3. World health statistics 2017: monitoring health for the SDGs, Sustainable Development Goals. Geneva: World Health Organization. 2017:1-116.

4. Genco RJ, Grossi SG, Ho A, Nishimura F, Murayama Y. A proposed model linking inflammation to obesity, diabetes, and periodontal infections. Journal of periodontology. 2005; 76:2075-84.

5. Instituto Colombiano de Bienestar Familiar (2015) Encuesta Nacional de la Situacion Nutricional en Colombia 2015. Bogotá: Instituto Colombiano de Bienestar Familiar.

6. Ard J. Obesity in the US: what is the best role for primary care? BMJ. 2015;350:g7846.

7. Suvan J, D'Aiuto F, Moles DR, Petrie A, Donos N. Association between overweight/obesity and periodontitis in adults. 
A systematic review. Obesity reviews: an official journal of the International Association for the Study of Obesity. 2011;12:e381-404.

8. Keller A, Rohde JF, Raymond K, Heitmann BL. Association between periodontal disease and overweight and obesity: a systematic review. Journal of periodontology. 2015;86:76676.

9. Flemmig TF. Periodontitis. Annals of periodontology / the American Academy of Periodontology. 1999;4:32-8.

10. Socransky SS, Haffajee AD. Periodontal microbial ecology. Periodontology. 2000 2005;38:135-87.

11. Papapanou PN. Periodontal diseases: epidemiology. Annals of periodontology / the American Academy of Periodontology. 1996;1:1-36.

12. Dyke TEV, Dave S. Risk Factors for Periodontitis. J Int Acad Periodontol. 2005 7:3-7.

13. Perlstein MI, Bissada NF. Influence of obesity and hypertension on the severity of periodontitis in rats. Oral Surg Oral Med Oral Pathol. 1977;43:707-19.

14. Khader YS, Bawadi HA, Haroun TF, Alomari M, Tayyem RF. The association between periodontal disease and obesity among adults in Jordan. Journal of clinical periodontology. 2009;36:18-24.

15. Al-Zahrani MS, Bissada NF, Borawskit EA. Obesity and periodontal disease in young, middle-aged, and older adults. Journal of periodontology. 2003;74:610-5.

16. Saito T, Shimazaki Y, Koga T, Tsuzuki M, Ohshima A. Relationship between upper body obesity and periodontitis. Journal of dental research. 2001;80:1631-6.

17. Saito T, Shimazaki Y, Kiyohara Y, et al. Relationship between obesity, glucose tolerance, and periodontal disease in Japanese women: the Hisayama study. J Periodontal Res. 2005;40:346-53.

18. Chaffee BW, Weston SJ. Association between chronic periodontal disease and obesity: a systematic review and metaanalysis. Journal of periodontology. 2010;81:1708-24.

19. Moura-Grec PG, Marsicano JA, Carvalho CA, Sales-Peres SH. Obesity and periodontitis: systematic review and metaanalysis. Ciencia \& saude coletiva. 2014;19:1763-72.

20. Akram Z, Abduljabbar T, Abu Hassan MI, Javed F, Vohra F. Cytokine Profile in Chronic Periodontitis Patients with and without Obesity: A Systematic Review and Meta-Analysis. Disease markers. 2016;2016:4801418.

21. OMS. El estado físico: uso e interpretación de la antropometría; Informe de un comité de expertos de la Organización Mundial de la Salud. Serie de informes técnicos, 854 GinebraSuiza. 1995.

22. Page RC, Eke PI. Case definitions for use in population-based surveillance of periodontitis. Journal of Periodontology. 2007;78:1387-99.

23. Eke PI, Page RC, Wei L, Thornton-Evans G, Genco RJ. Update of the case definitions for population-based surveillance of periodontitis. J Periodontol. 2012;83:1449-54.
24. Acunzo R, Limiroli E, Pagni G, Dudaite A, Consonni D, Rasperini G. Gingival Margin Stability After Mucogingival Plastic Surgery. The Effect of Manual Versus Powered Toothbrushing: A Randomized Clinical Trial. Journal of periodontology. 2016;87:1186-94.

25. Pataro AL, Costa FO, Cortelli SC, et al. Influence of obesity and bariatric surgery on the periodontal condition. Journal of Periodontology. 2012;83:257-66.

26. ENSAB, IV.Ministerio de Salud.2014. Disponible en: https:// www.minsalud.gov.co/sites/rid/Lists/BibliotecaDigital/ RIDE/VS/PP/ENSAB-IV-Situacion-Bucal-Actual.pdf. Consultado en: noviembre 1 de 2017.

27. Carvajal P, Gomez M, Gomes S, et al. Prevalence, severity, and risk indicators of gingival inflammation in a multi-center study on South American adults: a cross sectional study. Journal of applied oral science: revista FOB. 2016;24:524-34.

28. Zimmermann H, Zimmermann N, Hagenfeld D, Veile A, Kim TS, Becher H. Is frequency of tooth brushing a risk factor for periodontitis? A systematic review and meta-analysis. Community dentistry and oral epidemiology. 2015;43:116-27.

29. Position paper: epidemiology of periodontal diseases. American Academy of Periodontology. Journal of Periodontology. 1996;67:935-945.

30. de Castilhos ED, Horta BL, Gigante DP, Demarco FF, Peres KG, Peres MA. Association between obesity and periodontal disease in young adults: a population-based birth cohort. Journal of Clinical Periodontology. 2012;39:717-24.

31. Almerich-Silla JM, Alminana-Pastor PJ, Boronat-Catala M, Bellot-Arcis C, Montiel-Company JM. Socioeconomic factors and severity of periodontal disease in adults (35-44 years). A cross sectional study. Journal of Clinical and Experimental Dentistry. 2017;9:e988-e994.

32. Holde GE, Oscarson N, Trovik TA, Tillberg A, Jonsson B. Periodontitis Prevalence and Severity in Adults: A CrossSectional Study in Norwegian Circumpolar Communities. Journal of Periodontology. 2017;88:1012-22.

33. Figueiredo A, Soares S, Lopes H, et al. Destructive periodontal disease in adult Indians from Northeast Brazil: cross-sectional study of prevalence and risk indicators. Journal of Clinical Periodontology. 2013;40:1001-6.

34. Han K, Park JB. Age threshold for moderate and severe periodontitis among Korean adults without diabetes mellitus, hypertension, metabolic syndrome, and/or obesity. Medicine. 2017;96:e7835.

35. Boillot A, El Halabi B, Batty GD, Range H, Czernichow S, Bouchard P. Education as a predictor of chronic periodontitis: a systematic review with meta-analysis population-based studies. PloS one. 2011;6:e21508.

36. Martin-Cabezas R, Seelam N, Petit C, et al. Association between periodontitis and arterial hypertension: A systematic review and meta-analysis. American Heart Journal 2016;180:98-112. 
37. Pataro AL, Costa FO, Cortelli SC, Cortelli JR, Abreu MH, Costa JE. Association between severity of body mass index and periodontal condition in women. Clinical Oral Investigations. 2012;16:727-34.

38. Suresh S, Mahendra J. Multifactorial relationship of obesity and periodontal disease. Journal of Clinical and Diagnostic Research: JCDR. 2014;8:ZE01-03.
39. Goldberg RJ, McManus DD, Allison J. Greater knowledge and appreciation of commonly-used research study designs. The American Journal of Medicine. 2013;126:169 e161-8. 